\title{
Auranofin inhibits the proliferation of lung cancer cells via necrosis and caspase-dependent apoptosis
}

\author{
XIA YING CUI, SUN HYANG PARK and WOO HYUN PARK \\ Department of Physiology, Medical School, Research Institute for Endocrine Sciences, \\ Jeonbuk National University, Jeonju, Jeollabuk 54907, Republic of Korea
}

Received May 6, 2020; Accepted September 1, 2020

DOI: $10.3892 /$ or.2020.7818

\begin{abstract}
Auranofin, an inhibitor of thioredoxin reductase (TrxR), inhibits the growth of a variety of cancer cells. In the present study, various lung cancer cells were used to investigate the molecular basis of anti-cancer effects of auranofin, including cell death via apoptosis or necrosis and cell cycle arrest. Generally, auranofin inhibited the growth of the tested lung cancer cell lines in a dose-dependent manner with an IC50 of 3-4 $\mu \mathrm{M}$ at $24 \mathrm{~h}$. This agent significantly decreased the activity of TrxR in Calu-6 and A549 lung cancer cells. In addition, auranofin (3-5 $\mu \mathrm{M})$ triggered necrosis in lung cancer cells measured by the release of lactate dehydrogenase (LDH) into culture media. Auranofin increased the percentages of sub-G1 cells in Calu- 6 and A549 cells. DNA flow cytometry showed that auranofin induced G2/M phase arrest of Calu- 6 cells. This agent also efficiently induced apoptosis, accompanied by loss of mitochondrial membrane potential (MMP; $\Delta \Psi \mathrm{m})$, increases in cleavage forms of caspase-3 and poly (ADP-ribose) polymerase (PARP), and a high ratio of $\mathrm{BAX}$ to $\mathrm{Bcl}-2$ proteins. Furthermore, various caspase inhibitors reduced apoptosis and MMP $(\Delta \Psi \mathrm{m})$ loss in auranofin-treated Calu- 6 cells. In particular, the pan-caspase
\end{abstract}

Correspondence to: Professor Woo Hyun Park, Department of Physiology, Medical School, Research Institute for Endocrine Sciences, Jeonbuk National University, Deokjin, JeonJu, Jeollabuk 54907, Republic of Korea

E-mail: parkwh71@jbnu.ac.kr

Abbreviations: Trx, thioredoxin; TrxR, thioredoxin reductase; NSCLC, non-small cell lung cancer; SCLC, small cell lung cancer; MMP, mitochondrial membrane potential $(\Delta \Psi \mathrm{m})$; PARP, anti-poly ADP-ribose polymerase; FITC, fluorescein isothiocyanate; LDH, lactate dehydrogenase; MTT, 3-(4,5-dimethylthiazol-2-yl)-2,5diphenyltetrazolium bromide; Z-VAD, benzyloxycarbonyl-Val-AlaAsp-fluoromethylketone; Z-DEVD, benzyloxycarbonyl-Asp-GluVal-Asp-fluoromethylketone; Z-IETD, benzyloxycarbonyl-Ile-GluThr-Asp-fluoromethylketone; Z-LEHD, benzyloxycarbonyl-LeuGlu-His-Asp-fluoromethylketone

Key words: auranofin, lung cancer, thioredoxin reductase, apoptosis, necrosis, caspase inhibitor, benzyloxycarbonyl-Val-Ala-Asp-fluoromethylketone (Z-VAD), decreased cleavage forms of caspase-3, -8, and -9 in these cells. In conclusion, auranofin inhibited the proliferation of lung cancer cells, especially Calu- 6 cells, via cell cycle arrest and cell death due to necrosis or caspase-dependent apoptosis.

\section{Introduction}

Lung cancer is the leading cause of cancer-related death worldwide (1). Lung cancer is classified into two major types; small cell lung cancer (SCLC) accounting for 10-14\% of all lung cancer cases and non-SCLC (NSCLC) representing 85-90\% of all lung cancer cases (2). NSCLC is further divided into three subtypes according to histology: Squamous-cell carcinoma, adenocarcinoma, and large cell carcinoma (3). Various clinical cancer therapies have been used to treat lung cancer, but better efficacy is still required. Many studies report inhibition of cell growth and induction of apoptosis by many therapeutic agents $(4,5)$, yet novel agents that target specific intracellular targets of lung cancer cells continue to be developed.

Apoptosis is a cellular response to anti-cancer drugs. The mechanism of apoptosis mainly involves mitochondrial and cell death receptor pathways (6). The key element in the mitochondrial pathway is the efflux of cytochrome $\mathrm{c}$ from mitochondria to cytosol. In the cytosol, cytochrome c forms a complex (apoptosome) with apoptotic protease-activating factor 1 (Apaf-1) and caspase-9, leading to the activation of caspase-3 $(6,7)$. The induction of apoptosis is accompanied by increasing BAX and decreasing Bcl-2 levels, leading to the loss of mitochondrial membrane potential (MMP; $\Delta \Psi \mathrm{m})(8)$. The cell death receptor pathway is characterized by the binding of cell death ligands to their death receptors with subsequent activation of caspase- 8 and -3 (9). Caspase- 3 is a major executioner caspase, whose activation can systematically dismantle cells by cleaving key proteins, especially poly (ADP-ribose) polymerase (PARP) (10). Thus, targeted inhibition of anti-apoptotic pathways is an attractive concept for the design of cancer treatments.

Auranofin, a thioredoxin reductase (TrxR) inhibitor, was initially used for oral therapy for rheumatoid arthritis (11). Originally, this agent was considered an anti-inflammatory drug (12). Thioredoxin ( $\operatorname{Trx}$ ) and TrxR make a coupled redox system, which plays a key role in maintaining redox reactions 
in biosynthetic pathways and controlling redox homeostasis. Trx, a redox regulatory protein, can be oxidized by reactive oxygen species (ROS). Oxidative stress due to either overproduction of ROS or accumulation thereof can initiate events that lead to cell death $(13,14)$. Trx and TrxR are overexpressed in numerous cancer cells including lung Cancer (15). Modulation of the Trx system is thus a promising target for cancer therapy (11). Trx and TrxR expression are upregulated by nuclear factor-erythroid 2 p45-related factor 2 (16). Inhibition of $\operatorname{Trx} R$ increases the efficacy of anti-cancer drugs in lung and colon cancer (17-19). Downregulation of Trx by suberoyl bis-hydroxamic acid is closely involved in lung cancer cell death (20). Auranofin also induces apoptosis in mesothelioma and cervical cancer cells via oxidative stress $(13,21)$.

Understanding of the anti-cancer effects of auranofin in lung cancer cells remains poor. In the present study, various lung cancer cells were used to investigate the molecular basis of anti-cancer effects of auranofin, including cell death via apoptosis or necrosis and cell cycle arrest.

\section{Materials and methods}

Cell culture. Human SCLC cell line (Calu-6), adenocarcinoma cell lines (A549, SK-LU-1), and large cell carcinoma cell lines (NCI-H460, NCI-H1299) were obtained from the American Type Culture Collection (Manassas, VA). Normal human pulmonary fibroblast (HPF) cells were obtained from PromoCell GmbH (C-12360, Heidelberg, Germany). These cells were maintained in an incubator containing $5 \% \mathrm{CO}_{2}$ at $37^{\circ} \mathrm{C}$. HPF and lung cancer cells were cultured in RPMI-1640 containing 10\% fetal bovine serum (Sigma-Aldrich Co., St. Louis, MO) and 1\% penicillin-streptomycin (Gibco BRL, Grand Island, NY). Cells were grown in $100 \mathrm{~mm}$ plastic cell culture dishes (BD Falcon. Franklin Lakes, NJ) and harvested with trypsin-EDTA (Gibco BRL). HPF cells were used between passages of four to five.

Reagents. Auranofin was purchased from Sigma-Aldrich Co. and was dissolved in dimethyl sulfoxide (DMSO; Sigma-Aldrich Co.) at $10 \mathrm{mM}$ as a stock solution. Pan-caspase inhibitor benzyloxycarbonyl-Val-Ala-Asp-fluoromethylketone (Z-VAD-FMK), caspase-3 inhibitor benzyloxycarbonyl-AspGlu-Val-Asp-fluoromethylketone (Z-DEVD-FMK), caspase-8 inhibitor benzyloxycarbonyl-Ile-Glu-Thr-Aspfluoromethylketone (Z-IETD-FMK), and caspase-9 inhibitor benzyloxycarbonyl-Leu-Glu-His-Aspfluoromethylketone (Z-LEHD-FMK) were obtained from R\&D Systems, Inc. (Minneapolis, MN,) and dissolved in $10 \mathrm{mM}$ DMSO as stock solutions. Cells were pretreated with $15 \mu \mathrm{M}$ of individual caspase inhibitors for $1 \mathrm{~h}$ prior to the addition of auranofin. DMSO (0.01\%) was used as a control vehicle and did not affect cell growth or cell death.

Cell growth inhibition assay. The effects of auranofin on the proliferation of HPF and lung cancer cells were determined by 3-(4,5-dimethylthiazol-2-yl)-2,5-diphenyltetrazolium bromide (MTT, Sigma-Aldrich Co.) assays. Briefly, $3 \times 10^{4}$ cells were seeded into 96-well microtiter plates (Nunc). After incubation with the indicated doses of auranofin for $24 \mathrm{~h}, 20 \mu \mathrm{l}$ of MTT solution [2 $\mathrm{mg} / \mathrm{ml}$ in phosphate-buffered saline (PBS; GIBCO
BRL)] was added to each well. The plates were incubated for $4 \mathrm{~h}$ at $37^{\circ} \mathrm{C}$. Medium in plates was removed by pipetting, and 100-200 $\mu 1$ of DMSO was added to each well to solubilize formazan crystals. Optical density was measured at $570 \mathrm{~nm}$ using a microplate reader (Synergy ${ }^{\mathrm{TM}} 2$, BioTekR Instruments Inc. Winooski, VT).

Lactate dehydrogenase ( $L D H$ ) release assay. Necrosis in HPF and lung cancer cells treated with auranofin were evaluated by LDH kit (Sigma-Aldrich Co.) Briefly, $1 \times 10^{6}$ cells in $60 \mathrm{~mm}$ culture dishes (BD Falcon) were incubated with the indicated concentrations of auranofin for $24 \mathrm{~h}$. After treatment, cell culture media were collected and centrifuged for $5 \mathrm{~min}$ at $200 \mathrm{x}$ g at room temperature. Supernatants $(50 \mu \mathrm{l})$ were added to 96-lawell plates along with LDH assay reagent and incubated at room temperature for $30 \mathrm{~min}$. Absorbance values were measured at $490 \mathrm{~nm}$ using a microplate reader (Synergy ${ }^{\mathrm{TM}}$ 2). LDH release was expressed as the percentage of extracellular LDH activity compared with untreated control cells.

Cell cycle and sub-G1 cell analysis. Cell cycle and sub-G1 distributions of cells were determined by propidium iodide (PI, Sigma-Aldrich Co.; Ex/Em=488 nm/617 nm) staining, as previously described (21). Briefly, $1 \times 10^{6}$ cells in $60 \mathrm{~mm}$ culture dishes (BD Falcon) were incubated with the indicated concentrations of auranofin for $24 \mathrm{~h}$. After washing whole cells including floating cells with PBS, cells were fixed in 70\% ethanol. These cells were washed with PBS twice and then incubated with PI $(10 \mu \mathrm{g} / \mathrm{ml})$ and RNase (Sigma-Aldrich) at $37^{\circ} \mathrm{C}$ for $30 \mathrm{~min}$. Proportions of cells in different phases of cell cycle or with sub-G1 DNA content were measured and analyzed with a FAC Star flow cytometer (BD Sciences, Franklin Lakes, NJ, USA).

Detection of apoptosis. Apoptosis was identified by staining with annexin V-fluorescein isothiocyanate (FITC, Life Technologies; Ex/Em=488/519 $\mathrm{nm}$ ), as previously described (22). Briefly, $110^{6}$ cells in $60 \mathrm{~mm}$ culture dishes (BD Falcon) were incubated with the indicated concentrations of auranofin for $24 \mathrm{~h}$ with or without individual caspase inhibitors. Cells were washed twice with cold PBS and then suspended in $200 \mu \mathrm{l}$ of binding buffer $(10 \mathrm{mM}$ HEPES/NaOH $\mathrm{pH} 7.4,140 \mathrm{mM} \mathrm{NaCl}, 2.5 \mathrm{mM} \mathrm{CaCl} 2$ ) at a concentration of $5 \times 10^{5}$ cells $/ \mathrm{ml}$ at $37^{\circ} \mathrm{C}$ for $30 \mathrm{~min}$. Annexin V-FITC $(2 \mu \mathrm{l})$ and PI $(1 \mu \mathrm{g} / \mathrm{ml})$ were added, and cells were analyzed with a FACStar flow cytometer (BD Sciences).

Measurement of mitochondrial membrane potential $(\Delta \Psi \mathrm{m})$. The mitochondrial membrane potential (MMP, $\Delta \Psi \mathrm{m}$ ) was monitored using a fluorescent dye Rhodamine 123 (Sigma-Aldrich Co.; Ex/Em=485/535 nm), a cell-permeable cationic dye, which preferentially enters into mitochondria of their typical highly negative MMP $(\Delta \Psi \mathrm{m})$. Depolarization of MMP $(\Delta \Psi \mathrm{m})$ results in the loss of Rhodamine 123 from the mitochondria and decreases the intracellular fluorescence of this dye, as previously described (23). In brief, $1 \times 10^{6}$ cells in $60 \mathrm{~mm}$ culture dishes (Nunc) were incubated with the designated doses of auranofin for $24 \mathrm{~h}$ with or without $15 \mu \mathrm{M}$ individual caspase inhibitors. Cells were washed twice with PBS and incubated with Rhodamine $123(0.1 \mathrm{mg} / \mathrm{ml})$ at a 
A

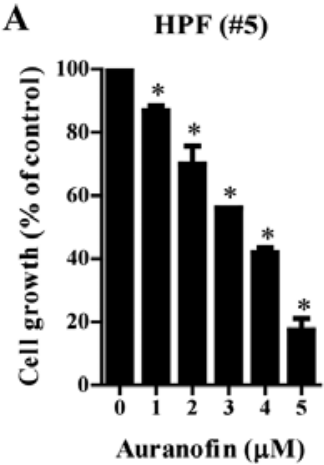

D

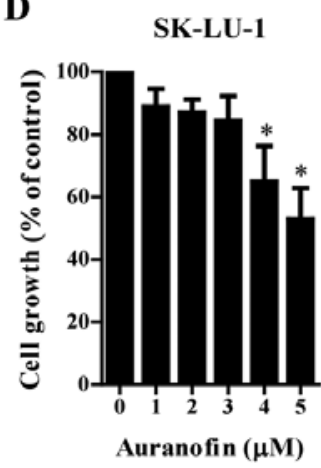

B

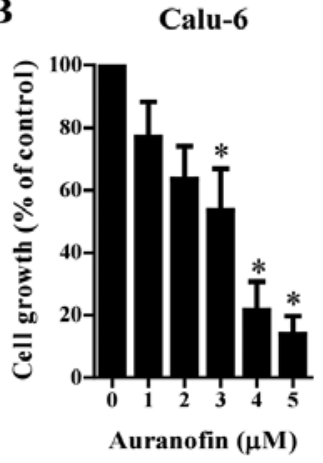

E

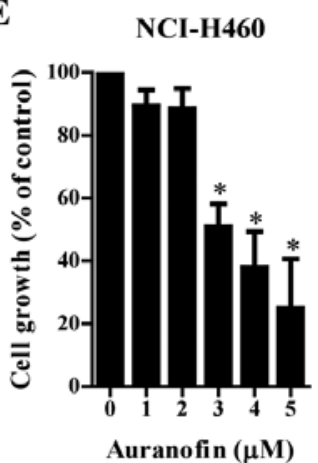

C

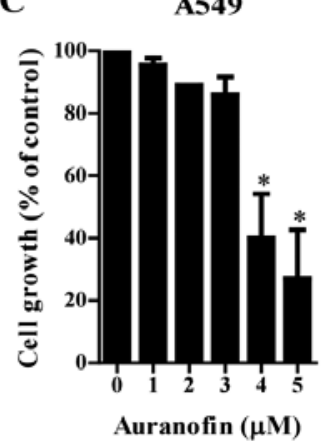

F

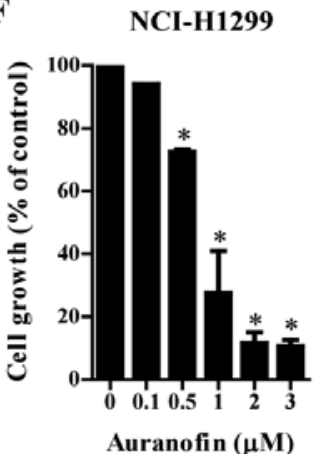

G

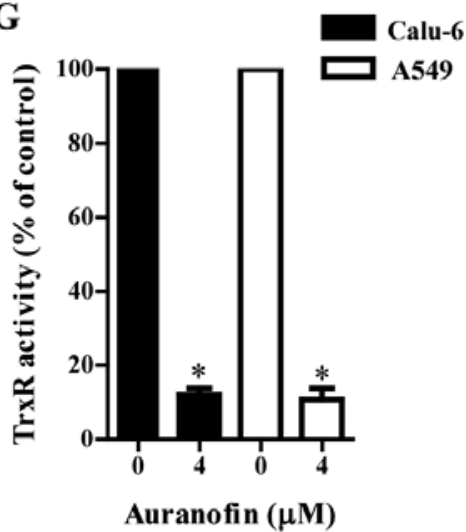

Figure 1. Effects of auranofin on cell growth and TrxR activity in lung cancer cells. Exponentially growing cells were incubated in the presence of the indicated concentrations of auranofin for $24 \mathrm{~h}$. Cell growth was assessed by MTT assays. (A-F) Graphs show cell growth in the normal HPF (A) and lung cancer cells, including Calu-6 (B), A549 (C), SK-LU-1 (D), NCI-H460 (E), and NCI-H1299 (F). (G) Graph shows the TrxR activity in Calu-6 and A549 cells. "P<0.05 compared with auranofin-untreated control cells.

concentration of $5 \times 10^{5}$ cells $/ \mathrm{ml}$ at $37^{\circ} \mathrm{C}$ for $30 \mathrm{~min}$. Rhodamine 123 staining intensities were determined using a FACStar flow cytometer. Rhodamine 123 negative (-) cells indicated MMP ( $\left.\Delta \Psi_{\mathrm{m}}\right)$ loss.

Western blot analysis. The protein expression levels were evaluated by western blotting. Briefly, $5 \times 10^{6}$ cells in $100 \mathrm{~mm}$ culture dishes (BD Falcon) were incubated condition with the indicated concentrations of auranofin at $37^{\circ} \mathrm{C}$ for $24 \mathrm{~h}$ with or without pan-caspase inhibitor, (Z-VAD). Cells were washed with PBS and lysed for $30 \mathrm{~min}$ in RIPA buffer supplemented with protease and phosphatase inhibitor cocktail (Intron Biotechnology, Seongnam Korea). The samples were heated to $100^{\circ} \mathrm{C}$ for $5 \mathrm{~min}$ and placed on ice. Total proteins $(30 \mu \mathrm{g})$ were resolved using 8-15\% SDS-PAGE gels and then transferred to Immobilon-P PVDF membranes (Millipore) by electroblotting. Membranes were probed with anti-PARP (no. 9543, 1:1,000 dilution), anti-cleaved PARP (no. 9541, 1:1,000 dilution), anti-caspase-3 (no. 9662, 1:1,000 dilution), anti-caspase-8 (no. 9746, 1:1,000 dilution), anti-caspase-9 (no. 9502, 1:1,000 dilution), anti-cleaved caspase-3 (no. 9661, 1:1,000 dilution), anti-cleaved caspase-8 (no. 9496, 1:1,000 dilution), anti-cleaved caspase-9 (no. 9501, 1:1,000), anti-Bcl-2 (no. 2872, 1:1,000 dilution), anti-BAX (no. 2774, 1:1,000 dilution) (Cell Signaling Technology); anti-Trx1 (SC-20146, 1:1,000 dilution) and anti-GAPDH (SC-25778, 1:1,000 dilution) (Santa Cruz Biotechnology). Membranes were incubated with horseradish peroxidase-conjugated secondary antibodies (Santa Cruz Biotechnology) at $4^{\circ} \mathrm{C}$ for $1 \mathrm{~h}$. Blots were developed using an EZ-Western Lumi Pico ECL solution kit (DoGen Bio
Co, Seoul, Korea). All band intensities were quantified using the Image J program (Fuji Film, Tokyo, Japan).

Detection of TrxR activity. The activity of Trx R was assessed using the Thioredoxin Reductase assay kit according to the manufacturer's instructions (Sigma-1Aldrich). In brief, $1 \times 10^{6}$ cells were incubated in $60 \mathrm{~mm}$ culture dish (Nunc) with the indicated dose of auranofin for $24 \mathrm{~h}$. The cells were then washed in PBS and suspended in five volumes of lysis buffer. Protein concentrations were determined using the Bradford method. Supernatant samples containing $30 \mu \mathrm{g}$ total protein were used for the determination of TrxR activity. These were added to each well in 96-well microtiter plates (Nunc) with $5,5^{\prime}$-dithiobis (2-nitrobenzoic) acid at $25^{\circ} \mathrm{C}$ for $1 \mathrm{~h}$. The optical density of each well was measured at $412 \mathrm{~nm}$ using a microplate reader (Synergy ${ }^{\mathrm{TM}}$ 2).

Statistical analysis. The results are reported as the mean of at least two or three independent experiments (mean \pm SD). Data were analyzed using Instat software (GraphPad Prism5). The Student's t-test or one-way analysis of variance with post-hoc analysis using Tukey's multiple comparison test was used for the parametric data. Statistical significance was defined as $\mathrm{P}<0.05$.

\section{Results}

Effects of auranofin on cell growth and TrxR activity in lung cancer cells. The effect of auranofin, a known inhibitor of TrxR, on the growth of normal lung cell and lung cancer cell types was examined using MTT assays. The growth of normal HPF 
A

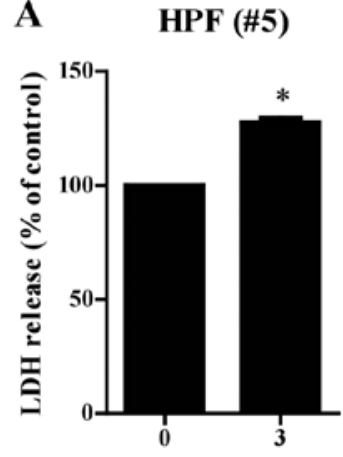

Auranofin $(\mu \mathrm{M})$

D

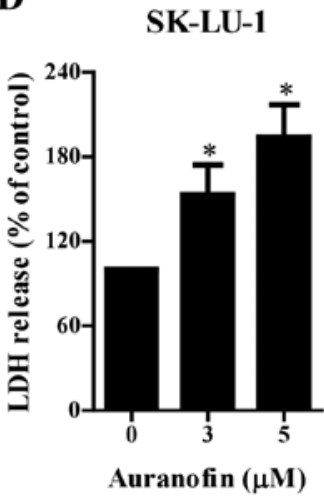

B

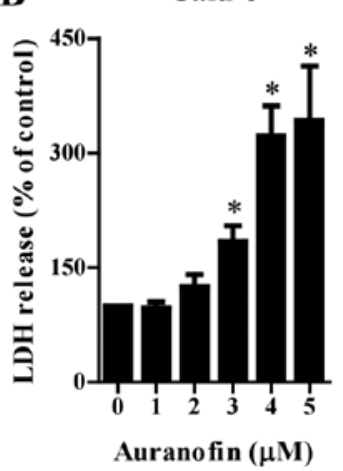

$\mathbf{E}$

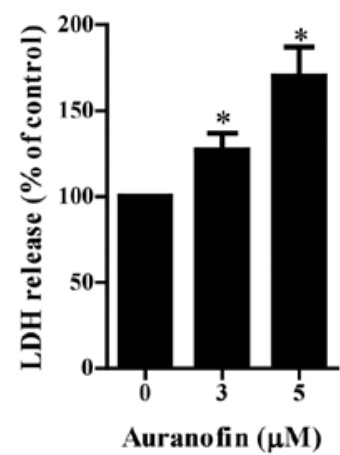

C

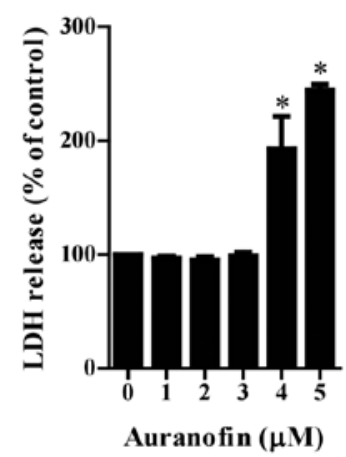

$\mathbf{F}$

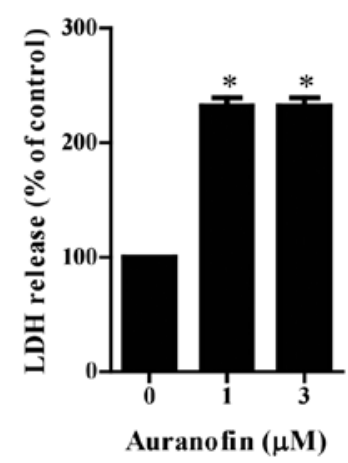

Figure 2. Effects of auranofin on necrosis in lung cancer cells. Exponentially growing cells were incubated in the presence of the designated concentrations of auranofin for $24 \mathrm{~h}$. Graphs show LDH releases in the normal HPF (A) and lung cancer cells including Calu-6 (B), A549 (C), SK-LU-1 (D), NCI-H460 (E), and NCI-H1299 (F). "P<0.05 compared with the auranofin-untreated control group.

cells showed dose-dependent inhibition with an $\mathrm{IC}_{50}$ of $\sim 3 \mu \mathrm{M}$ (Fig. 1A) after a 24-h incubation with auranofin. The growth of Calu-6 cells was also dose-dependently reduced with an $\mathrm{IC}_{50}$ of $\sim 3 \mu \mathrm{M}$ (Fig. 1B). The growth of A549 and SK-LU-1 cells was marginally reduced by $1-3 \mu \mathrm{M}$ auranofin and significantly decreased by 4-5 $\mu \mathrm{M}$ auranofin (Fig. 1C and D). Auranofin inhibited the growth of NCI-H460 and NCI-H1299 cells in a dose-dependent manner with $\mathrm{IC}_{50}$ of $\sim 3 \mu \mathrm{M}$ (Fig. 1E) and $1 \mu \mathrm{M}$ (Fig. 1F). Furthermore, auranofin significantly decreased the activity of TrxR in Calu-6 and A549 cells (Fig. 1G). Auranofin also downregulated the expression of Trx 1 protein in Calu- 6 and A549 cells (Fig. S1).

Effects of auranofin on cell death in lung cancer cells. $\mathrm{LDH}$ release was measured to determine whether auranofin causes cell necrosis. Treatment increased the release of LDH in the normal HPF cells after a $24 \mathrm{~h}$ incubation with $3 \mu \mathrm{M}$ auranofin (Fig. 2A). Auranofin (3-5 $\mu \mathrm{M}$ ) induced significant LDH release in Calu-6, SK-LU-1, and NCI-H460 cells in a dose-dependent manner (Fig. 2B, D and E), at 4-5 $\mu \mathrm{M}$ triggered LDH release in A549 cells (Fig. 2C), and at 1 and $3 \mu \mathrm{M}$ concentrations increased LDH release in NCI-H1299 cells (Fig. 2F).

Effects of auranofin on the cell cycle distributions in Calu-6 and A549 lung cancer cells. As growth inhibition of Calu-6 and A549 cells by auranofin could be explained by an arrest during cell cycle progression, distribution of the cells in different stages of the cell cycle were examined after a $24 \mathrm{~h}$ incubation with auranofin. DNA flow cytometric analysis indicated that 2 and $3 \mu \mathrm{M}$ auranofin induced a $\mathrm{G} 2 / \mathrm{M}$ phase arrest of the cell cycle in Calu- 6 cells and that $1 \mu \mathrm{M}$ auranofin did not affect cell cycle distributions. In addition, auranofin did not show specific cell cycle arrest in A549 cells (Fig. 3A and B). Moreover, auranofin significantly increased the percentages of sub-G1 cells in Calu-6 and A549 cells at 24 h (Fig. 3B).

Effects of auranofin on apoptosis in lung cancer cells. Whether auranofin induces apoptosis in cells was assessed using an annexin V-staining assay. The number of annexin V-positive normal HPF and Calu- 6 cells significantly increased in a dose-dependent manner after treatment with 1-5 $\mu \mathrm{M}$ auranofin (Fig. 4A and B). At 4-5 $\mu \mathrm{M}$, the number of annexin V-positive A549 cells was greatly increased (Fig. 4C). Similarly, treatment with 1-5 $\mu \mathrm{M}$ auranofin increased the number of annexin V-positive SK-LU-1 cells (Fig. 4D). The number of annexin V-positive NCI-H460 and NCI-H1299 cells were increased after incubation in 3-5 and 1-3 $\mu \mathrm{M}$ concentrations of auranofin, respectively (Fig. 4E and F).

Effects of auranofin on mitochondrial membrane potential $(M M P ; \Delta \Psi m)$ in lung cancer cells. Since apoptosis is closely related to the collapse of MMP $(\Delta \Psi \mathrm{m})$, loss of MMP $(\Delta \Psi \mathrm{m})$ in auranofin-treated cells was assessed using Rhodamine 123 dye. Loss of MMP $(\Delta \Psi \mathrm{m})$ in the normal HPF cells was dose-dependently induced by auranofin at concentrations of 2-5 $\mu \mathrm{M}$ (Fig. 5A). Similar loss of MMP ( $\Delta \Psi \mathrm{m})$ was observed 

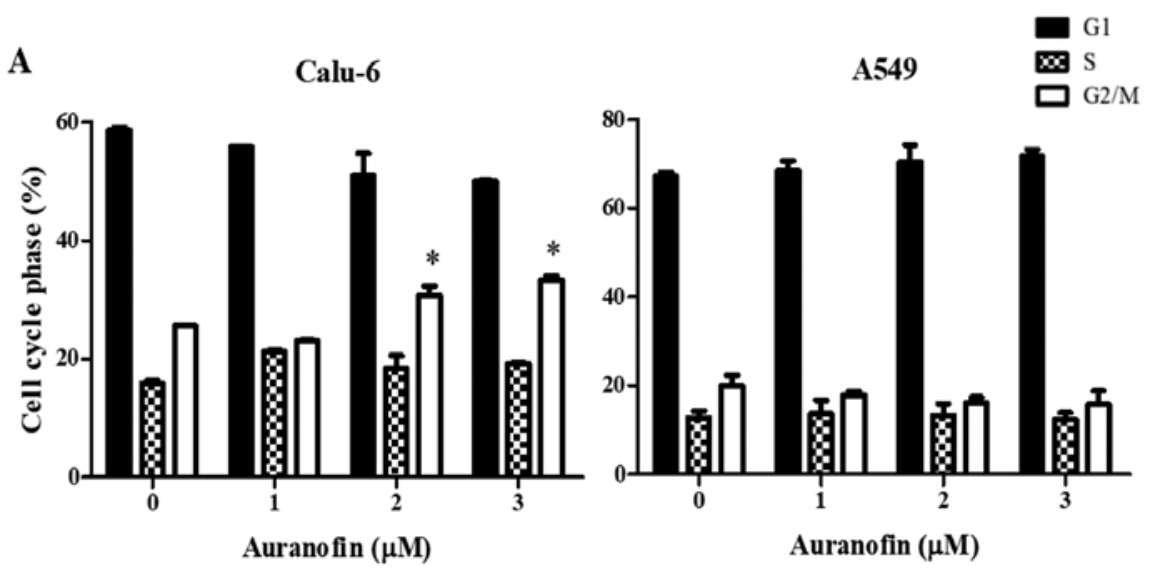

B
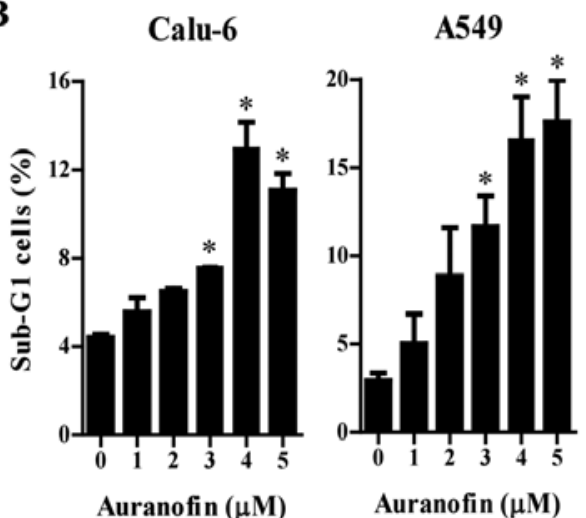

Figure 3. Effects of auranofin on cell cycle phase distributions in Calu-6 and A549 cells. Exponentially growing cells were incubated in the presence of the indicated concentrations of auranofin for $24 \mathrm{~h}$. Cell cycle phase distributions were assessed by DNA flow cytometry. (A) Graphs show cell cycle phase distributions in Calu- 6 and A549 cells. (B) Graphs show the percentages of sub-G1 cells in Calu- 6 and A549 cells. "P $<0.05$ compared with auranofin-untreated control cells.

A

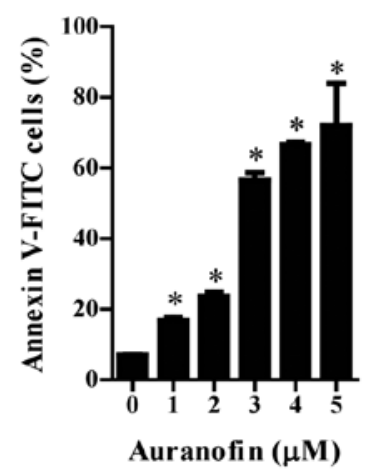

D

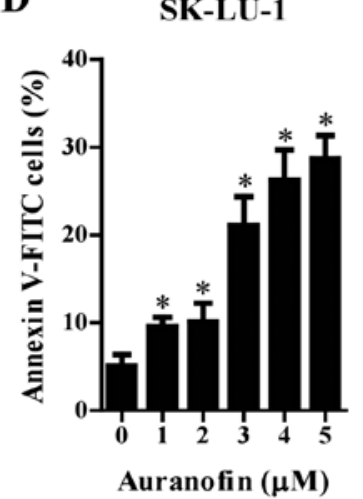

B

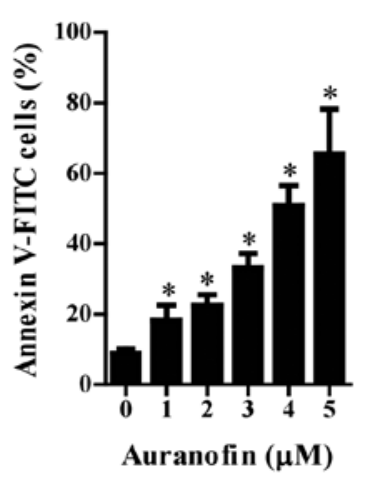

$\mathbf{E}$

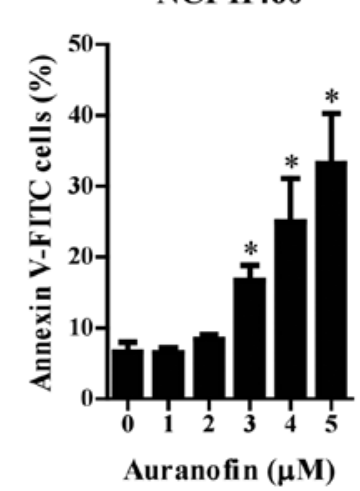

C

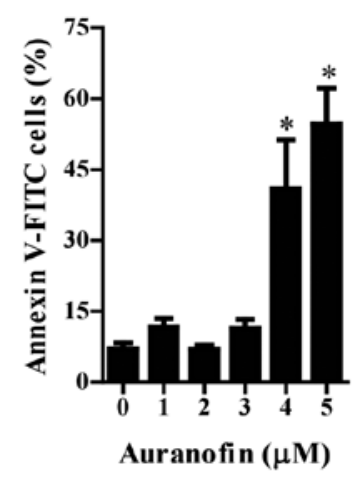

F

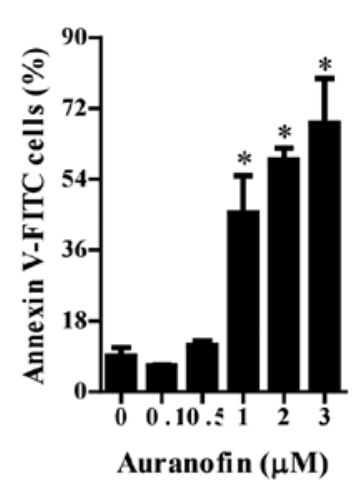

Figure 4. Effects of auranofin on apoptosis in lung cancer cells. Exponentially growing cells were incubated in the presence of the indicated concentrations of auranofin for $24 \mathrm{~h}$. Annexin V-stained cells were measured with FACStar flow cytometer. Graphs show the percentages of annexin V-stained cells in the normal HPF (A) and lung cancer cells, Calu-6 (B), A549 (C), SK-LU-1 (D), NCI-H460 (E), and NCI-H1299 (F). "P<0.05 compared with auranofin-untreated control cells.

after treatment of Calu-6 and SK-LU-1 cells (Fig. 5B and D), A549 cells (Fig. 5C), and NCI-H460 cells (Fig. 5E) with auranofin at 3-5, 4-5, and 2-5 $\mu \mathrm{M}$, respectively. Concentrations of 1-3 $\mu \mathrm{M}$ auranofin did not show this effect in A549 cells (Fig. 5C). Furthermore, auranofin at concentrations of 0.1-0.5 $\mu \mathrm{M}$ significantly increased loss of MMP $(\Delta \Psi \mathrm{m})$ in NCI-H1299 cells (Fig. 5F).
Effects of auranofin on apoptosis-related protein levels in Calu-6 and A549 cells. As auranofin increased the number of annexin V-positive cells, levels of apoptosis-related proteins were evaluated by western blot analysis. Intact forms of PARP decreased in auranofin-treated Calu- 6 and A549 cells whereas the cleavage forms of PARP increased in these cells (Fig. 6A and C). In addition, the levels of cleaved caspase-3 
A

HPF (\#5)

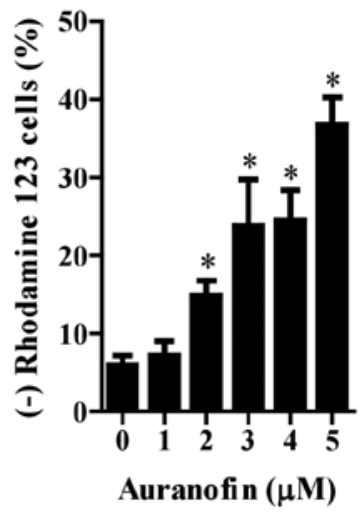

D

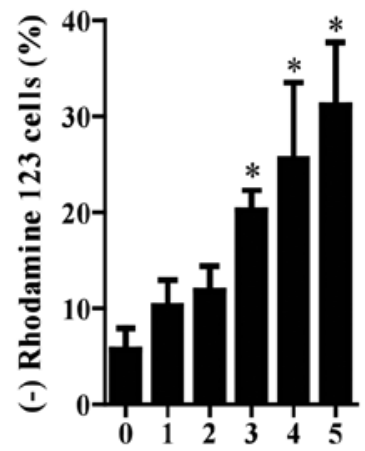

Auranofin $(\mu \mathrm{M})$
B

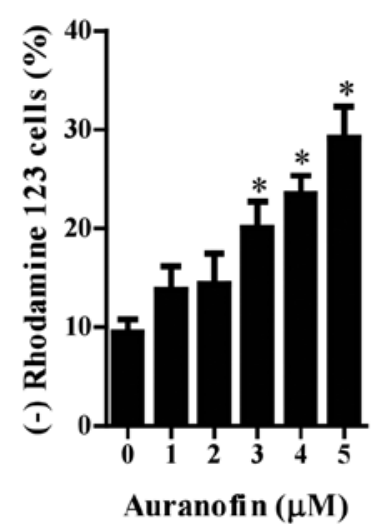

$\mathbf{E}$

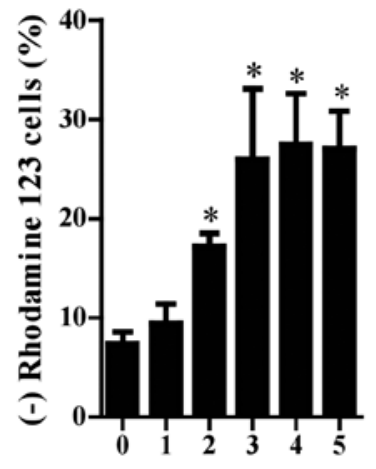

Auranofin $(\mu \mathrm{M})$
C

A549

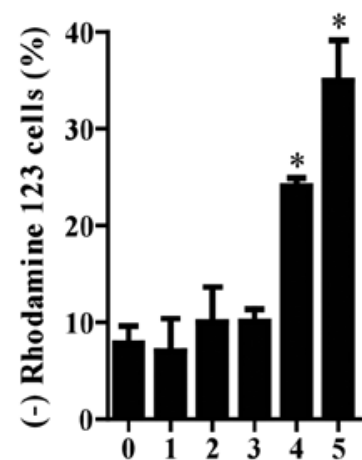

Auranofin $(\mu \mathbf{M})$

\section{F}

NCI-H1299

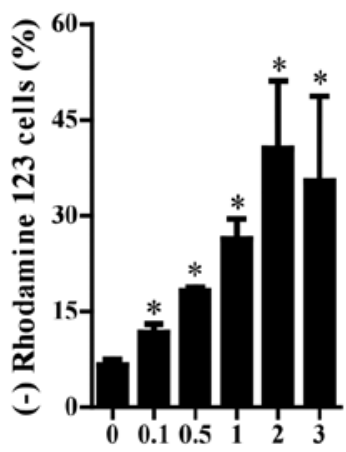

Auranofin $(\mu \mathrm{M})$

Figure 5. Effects of auranofin on mitochondrial membrane potential (MMP; $\Delta \Psi \mathrm{m}$ ) in lung cancer cells. Exponentially growing cells incubated in the presence of the indicated concentrations of auranofin for $24 \mathrm{~h}$. Percentages of Rhodamine 123-negative [MMP $(\Delta \Psi \mathrm{m})$ loss] cells were measured using FACStar flow cytometer. Graphs show MMP $(\Delta \Psi \mathrm{m})$ loss cells in normal HPF (A) and lung cancer cells, including Calu-6 (B), A549 (C), SK-LU-1 (D), NCI-H460 (E), and NCI-H1299 (F). ${ }^{*} \mathrm{P}<0.05$ compared with auranofin-untreated control cells.

A

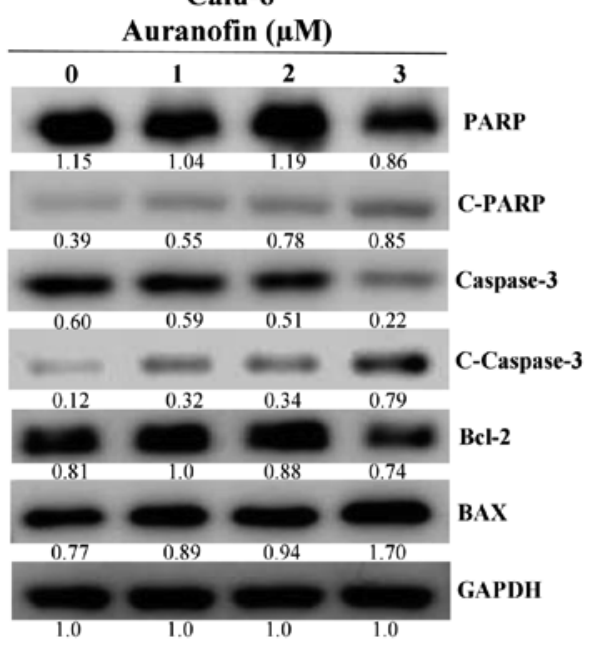

B

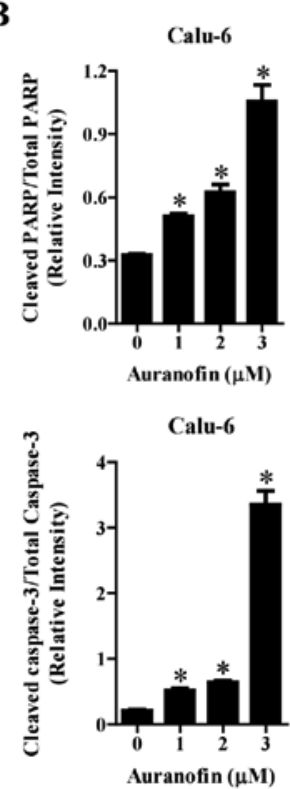

C

A549
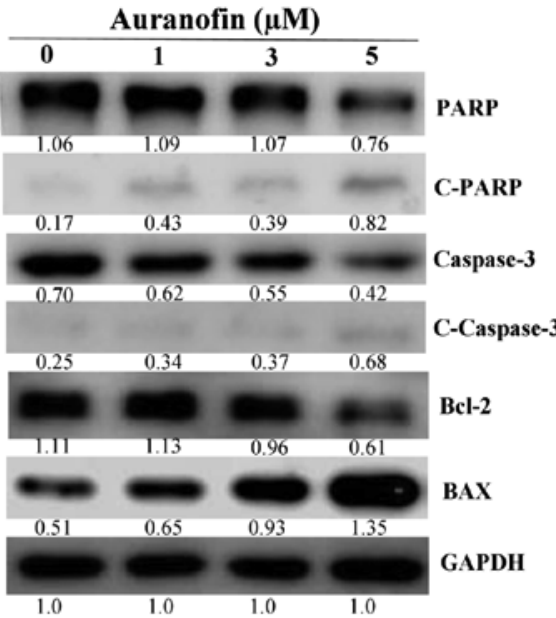

D
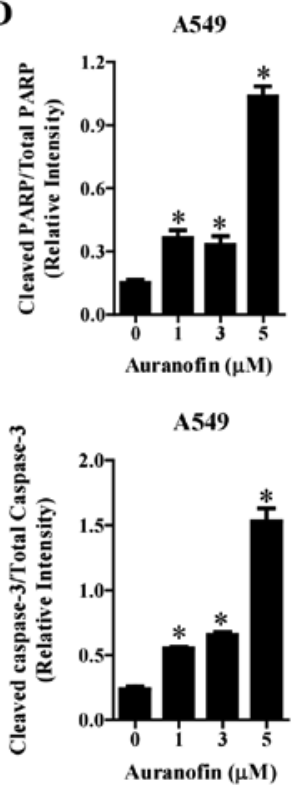

Figure 6. Effects of auranofin on apoptosis-related protein levels in Calu-6 and A549 cells. Exponentially growing cells were incubated in the presence of the indicated concentrations of auranofin for $24 \mathrm{~h}$. Protein extracts were resolved by 8-15\% SDS-PAGE gel, transferred to PVDF membranes, and immunoblotted with the indicated antibodies. Western blot analysis shows the levels of PARP, cleaved PARP, cleaved caspase-3, Bcl-2, BAX, and GAPDH in auranofin-treated Calu-6 (A) and A549 cells (C). Graphs show ratio of Cleaved PARP/Total PARP and Cleaved caspase-3/Total caspase-3 in auranofin-treated Calu-6 (B) and A549 (D) cells, and band intensities were quantified using the Image J program. ${ }^{*} \mathrm{P}<0.05$ compared with auranofin-untreated control cells. 

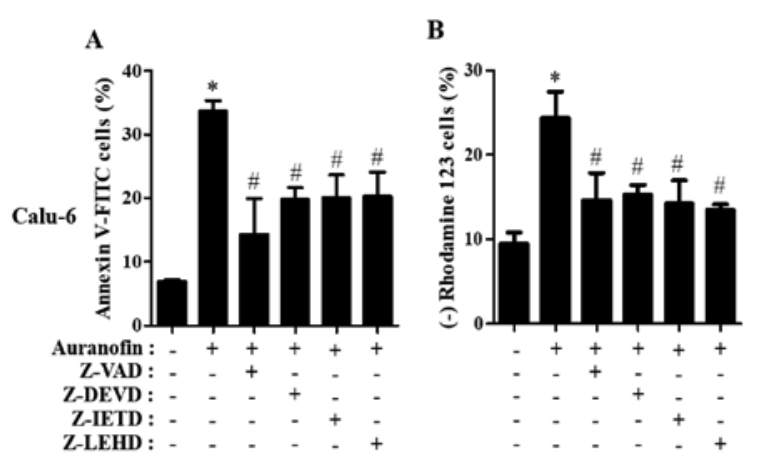

E

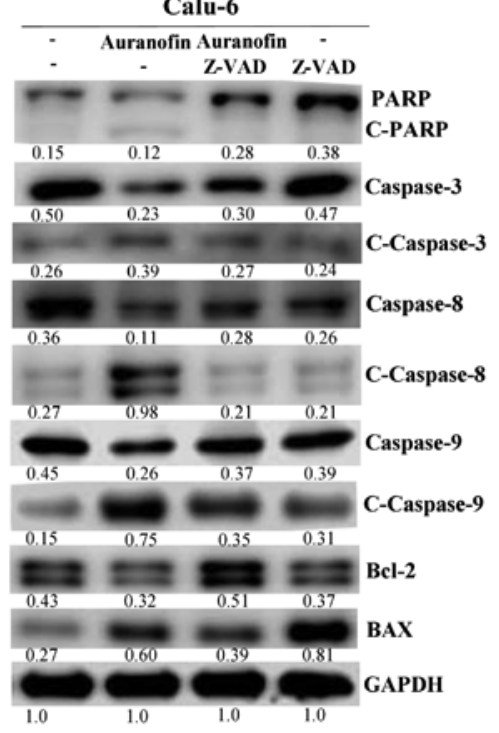

$\mathbf{F}$
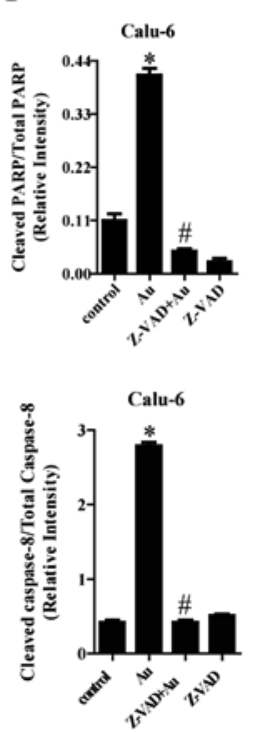

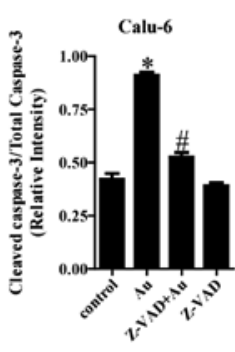

D

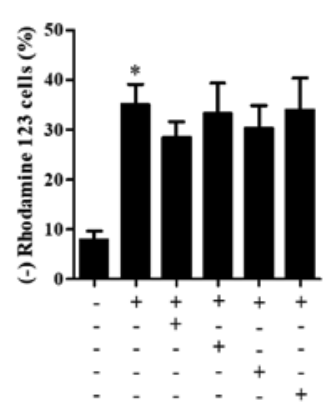

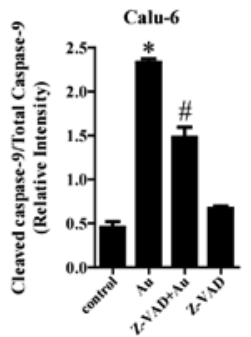

Figure 7. Effects of caspase inhibitors on cell death, MMP $(\Delta \Psi \mathrm{m})$ loss, and apoptosis-related proteins in auranofin-treated cells. Exponentially growing Calu-6 and A549 cells were incubated in the presence of 3 and $5 \mu \mathrm{M}$ auranofin for $24 \mathrm{~h}$, respectively, following $1 \mathrm{~h}$ preincubation with $15 \mu \mathrm{M}$ of individual caspase inhibitors. Graphs show the percentages of annexin V-positive cells (A) and Rhodamine 123-negative [MMP ( $\triangle \Psi \mathrm{m})$ loss] cells as assessed using FACStar flow cytometer with Calu-6 (A and B) and A549 cells (C and D). Protein extracts were resolved by 8-15\% SDS-PAGE gel, transferred to PVDF membranes, and immunoblotted with the indicated antibodies. Western blot results show the levels of PARP; cleaved caspase-3, -8, -9; Bcl-2; BAX; and GAPDH (E). Graphs show ratio of Cleaved PARP/Total PARP, Cleaved caspase-3/Total caspase-3, Cleaved caspase-8/Total caspase-8 and Cleaved caspase-9/Total caspase-9 in auranofin-treated Calu- 6 cells (F), and band intensities were quantified using the Image J program. *P $<0.05$ compared with auranofin-untreated control cells. ${ }^{\#} \mathrm{P}<0.05$ compared with cells treated with auranofin only.

were dose-dependently upregulated in auranofin-treated Calu-6 and A549 cells (Fig. 6A and C). Auranofin also decreased the levels of Bcl-2 and increased the levels of BAX in Calu-6 and A549 cells (Fig. 6A and C). The ratios of cleaved PARP/total PARP and cleaved caspase-3/total caspase-3 were increased in auranofin-treated Calu- 6 and A549 cells (Fig. 6B and D). All blots presented together were probed from the same membrane.

Effects of caspase inhibitors on cell death, MMP ( $\Delta \Psi \mathrm{m})$, and apoptosis-related protein levels in auranofin-treated Calu- 6 and $A 549$ cells. To determine which caspases were involved in auranofin-induced apoptosis, cells were pretreated with various caspase inhibitors before treatment with auranofin. Z-VAD (a pan-caspase inhibitor) significantly decreased the number of annexin V-positive Calu-6 cells treated with $3 \mu \mathrm{M}$ auranofin (Fig. 7A). Furthermore, all of the tested caspase inhibitors (Z-DVED for caspase-3, Z-IETD for caspase-8, and Z-LEHD for caspase-9) significantly reduced the death of Calu- 6 cells following auranofin treatment (Fig. 7A). In addition, all caspase inhibitors significantly protected against the loss of MMP $(\Delta \Psi \mathrm{m})$ in Calu-6 cells caused by auranofin (Fig. 7B). Likewise, all tested caspase inhibitors slightly decreased apoptotic A549 cell death following incubation with $5 \mu \mathrm{M}$ auranofin (Fig. 7C). However, these decreases were not statistically significant. Caspase inhibitors marginally reduced the loss of MMP $(\Delta \Psi \mathrm{m})$ in auranofin-treated A549 cells (Fig. 7D). The expression of apoptosis-related proteins showed an increase in the intact form of PARP in auranofin-treated Calu- 6 cells in the presence of Z-VAD and a decrease in the cleavage form of PARP in those cells (Fig. 7E). Furthermore, Z-VAD reduced cleavage forms of caspase-3, -8 , and -9 in auranofin-treated Calu- 6 cells (Fig. 7E). Finally, the expression of Bcl-2 in auranofin-treated cells was clearly upregulated in the presence of Z-VAD, and the levels of BAX in those cells were downregulated (Fig. 7E). The ratio of cleaved PARP/total PARP, cleaved caspase-3/total caspase-3, cleaved caspase-8/total caspase- 8 and cleaved caspase-9/total caspase- 9 were increased in auranofin-treated Calu-6 cells (Fig. 7F). However, these were decreased in auranofin and Z-VAD treated Calu- 6 cells (Fig. 7F). All blots presented together were probed from the same membrane.

\section{Discussion}

Although auranofin was approved by the U.S. Food and Drug Administration for the treatment of rheumatoid arthritis, this agent has recently been studied as a possible therapeutic drug for various human diseases, including cancer (11). According to the current result, auranofin inhibited the activity of TrxR in Calu-6 and A549 cells, supporting that auranofin is a TrxR inhibitor. This study demonstrated that auranofin significantly and efficiently decreased the growth of lung cancer cells in a dose-dependent manner. The sensitivities of lung cancer cells to auranofin treatment are generally lower than those of prostate, leukemia, and ovarian cancer cell lines (24-26). However, they are similar to those of 


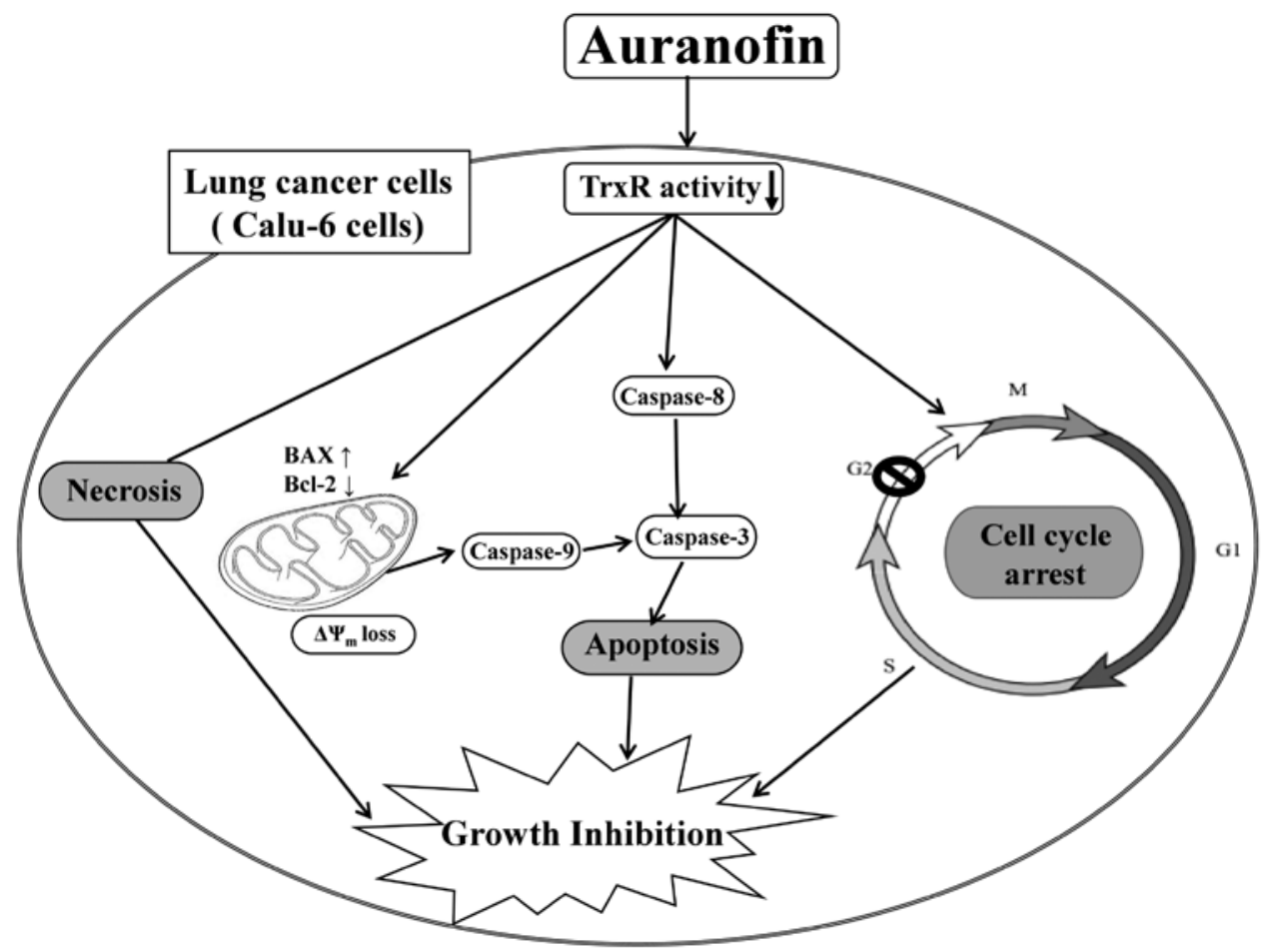

Figure 8. Schematic diagram of the anti-proliferation effects of auranofin on lung cancer cells, with a focus on Calu- 6 cells.

cervical cancer and mesothelioma cancer cell lines $(13,21)$ Interestingly, NCI-H1299 cell growth was inhibited by a lower dose of auranofin $(0.5 \mu \mathrm{M})$ after a $24 \mathrm{~h}$ incubation. This result suggests high sensitivity of these cells. The growth of normal HPF cells was dose dependently reduced by auranofin with an $\mathrm{IC}_{50}$ of approximately $3 \mu \mathrm{M}$. Survival and proliferation of TrxR1-deficient tumors strictly depend on a functional glutathione system (27). Those results suggest that sensitivity to auranofin depends on the varying capacity of antioxidation pathways in different cell types.

Auranofin induces apoptosis in normal and lung cancer cells. In particular, Calu-6 and A549 cells treated with auranofin appear to show a decrease in Bcl-2 levels and an increase in BAX levels, along with increases in the cleavage forms of caspase-3 and PARP. In addition, auranofin dose-dependently triggered necrosis in these cells, as evidenced by the release of LDH. This agent also increased the percentages of sub-G1 cells in Calu-6 and A549 cells. Thus, auranofin induced lung cancer cell death via apoptosis and/or necrosis, depending on its concentrations. DNA flow cytometry indicates that auranofin induced arrest at the G2/M phase of the cell cycle in Calu- 6 cells. Similarly, a TrxR-1 inhibitor, Chaetocin, induced G2/M phase arrest in gastric cancer cells (28). Thus, G2/M phase arrest is a plausible underlying mechanism for the inhibition of cell proliferation. Of note, auranofin led to G1 phase arrest in SK-LU-1 cells (data not shown) and, in A549 cells, auranofin did not induce arrest in any specific phase of the cell cycle. These results indicate that specificity of cell cycle arrest depends on both auranofin concentration and cell type. Use of auranofin for cancer therapy should be subject to consideration of the various mechanisms involved in the anti-cancer effects of auranofin as well as the specificity of cells in the target tumor.
Apoptosis is closely associated with the collapse of MMP $(\Delta \Psi \mathrm{m})$, and auranofin can cause a breakdown in MMP $(\Delta \Psi \mathrm{m})$ (29). Similarly, auranofin induced the loss of MMP $(\Delta \Psi \mathrm{m})$ in both normal and lung cancer cells. The degree of MMP $(\Delta \Psi \mathrm{m})$ loss in auranofin-treated lung cells was very similar to that of annexin V-positive cells. For example, concentrations of 1-3 $\mu \mathrm{M}$ auranofin that did not induce apoptosis in A549 cells also did not significantly increase the loss of MMP $(\Delta \Psi \mathrm{m})$. Interestingly, although lower doses of auranofin did not induce apoptosis in large cell carcinoma cells (NCI-H460 and NCI-H1299), such doses did trigger the loss of MMP $(\Delta \Psi \mathrm{m})$. These results suggest that auranofin initially impacts mitochondrial membranes, especially large cell carcinoma cells, which precedes the next step in apoptosis. Additionally, differences in sensitivity to auranofin in relation to $\mathrm{MMP}(\Delta \Psi \mathrm{m})$ and apoptosis are probably due to the different basal activities of mitochondria, which vary by cell type, tissue origin, and species (30).

Apoptosis involves cell death receptor (extrinsic) and mitochondrial (intrinsic) pathways (6). When auranofin-treated Calu-6 and A549 cells were treated with various caspase inhibitors, these inhibitors, including Z-VAD, significantly decreased the percentages of annexin V-stained Calu-6 cells and MMP $(\Delta \Psi \mathrm{m})$ loss following auranofin treatment in cells. In addition, Z-VAD reduced cleavage forms of caspase-3, -8, and -9 in these cells, upregulated the expression of Bcl-2, and downregulated the levels of BAX. All caspase inhibitors decreased to some extent the numbers of annexin $\mathrm{V}$-stained A549 cells and MMP $(\Delta \Psi \mathrm{m})$ loss following auranofin treatment. Thus, auranofin-induced apoptosis in lung cancer cells may involve both extrinsic and intrinsic pathways.

In conclusion, auranofin efficiently inhibits lung cancer cell proliferation, especially in Calu- 6 cells. This inhibition is 
mediated by cell cycle arrest and cell death due to necrosis and caspase-dependent apoptosis (Fig. 8). The present data provide useful information for understanding cellular and molecular anti-cancer mechanisms of auranofin in lung cancer cells.

\section{Acknowledgements}

Not applicable.

\section{Funding}

The present study was supported by 'Research Base Construction fund Support Program' funded by Jeonbuk National University in 2020 and the Basic Science Research Program through the National Research Foundation of Korea (NRF) funded by the Ministry of Education (2019R1I1A2A01041209).

\section{Availability of data and materials}

Data collected during the present study are available from the corresponding author upon reasonable request.

\section{Authors' contributions}

WHP and XYC designed the study. XYC mainly conducted experiments and wrote early version of the manuscript. Specifically, XYC retained cells and performed flow cytometry. SHP assisted with providing resources for cell culture, flow cytometry, and other experiments. XYC and SHP completed statistical analysis. WHP and CXY reviewed and edited the final manuscript. All authors have read and approved the final version of the manuscript, and have verified that the accuracy and integrity of all parts of the work have been properly investigated and addressed.

\section{Ethics approval and consent to participate}

Not applicable.

\section{Patient consent for publication}

Not applicable.

\section{Competing interests}

The authors declare that they have no competing interests.

\section{References}

1. Hu Z, Li M, Chen Z, Zhan C, Lin Z and Wang Q: Advances in clinical trials of targeted therapy and immunotherapy of lung cancer in 2018. Transl Lung Cancer Res 8: 1091-1106, 2019.

2. Dela Cruz CS, Tanoue LT and Matthay RA: Lung cancer: Epidemiology, etiology, and prevention. Clin Chest Med 32: 605-644, 2011.

3. Carter BW, Lichtenberger JP III, Benveniste MK, de Groot PM, Wu CC, Erasmus JJ and Truong MT: Revisions to the TNM staging of lung cancer: Rationale, significance, and clinical application. Radiographics 38: 374-391, 2018.

4. Park HK, Han BR and Park WH: Combination of arsenic trioxide and valproic acid efficiently inhibits growth of lung cancer cells via G2/M-phase arrest and apoptotic cell death. Int J Mol Sci 21: 2649, 2020.
5. You BR and Park WH: Suberoyl bishydroxamic acid inhibits the growth of A549 lung cancer cells via caspase-dependent apoptosis. Mol Cell Biochem 344: 203-210, 2010.

6. Chung C: Restoring the switch for cancer cell death: Targeting the apoptosis signaling pathway. Am J Health Syst Pharm 75: 945-952, 2018

7. Würstle ML, Laussmann MA and Rehm M: The central role of initiator caspase-9 in apoptosis signal transduction and the regulation of its activation and activity on the apoptosome. Exp Cell Res 318: 1213-1220, 2012.

8. Huska JD, Lamb HM and Hardwick JM: Overview of BCL-2 family proteins and therapeutic potentials. Methods Mol Biol 1877: 1-21, 2019.

9. Liu X, Yue P, Zhou Z, Khuri FR and Sun SY: Death receptor regulation and celecoxib-induced apoptosis in human lung cancer cells. J Natl Cancer Inst 96: 1769-1780, 2004.

10. Hengartner MO: The biochemistry of apoptosis. Nature 407: $770-776,2000$.

11. Onodera T, Momose I and Kawada M: Potential anticancer activity of auranofin. Chem Pharm Bull (Tokyo) 67: 186-191, 2019.

12. Isakov E, Weisman-Shomer $\mathrm{P}$ and Benhar M: Suppression of the pro-inflammatory NLRP3/interleukin-1 $\beta$ pathway in macrophages by the thioredoxin reductase inhibitor auranofin. Biochim Biophys Acta 1840: 3153-3161, 2014.

13. You BR and Park WH: Auranofin induces mesothelioma cell death through oxidative stress and GSH depletion. Oncol Rep 35: 546-551, 2016.

14. Park WH and You BR: Antimycin A induces death of the human pulmonary fibroblast cells via ROS increase and GSH depletion. Int J Oncol 48: 813-820, 2016.

15. Fernandes AP, Capitanio A, Selenius M, Brodin O, Rundlöf AK and Björnstedt M: Expression profiles of thioredoxin family proteins in human lung cancer tissue: Correlation with proliferation and differentiation. Histopathology 55: 313-320, 2009.

16. Hawkes HJ, Karlenius TC and Tonissen KF: Regulation of the human thioredoxin gene promoter and its key substrates: A study of functional and putative regulatory elements. Biochim Biophys Acta 1840: 303-314, 2014

17. Yan X, Zhang X, Wang L, Zhang R, Pu X, Wu S, Li L, Tong P, Wang J, Meng QH, et al: Inhibition of thioredoxin/thioredoxin reductase induces synthetic lethality in lung cancers with compromised glutathione homeostasis. Cancer Res 79: 125-132, 2019.

18. Fu JN, Li J, Tan Q, Yin HW, Xiong K, Wang TY, Ren XY and Zeng HH: Thioredoxin reductase inhibitor ethaselen increases the drug sensitivity of the colon cancer cell line LoVo towards cisplatin via regulation of $\mathrm{G} 1$ phase and reversal of $\mathrm{G} 2 / \mathrm{M}$ phase arrest. Invest New Drugs 29: 627-636, 2011.

19. Poerschke RL and Moos PJ: Thioredoxin reductase 1 knockdown enhances selenazolidine cytotoxicity in human lung cancer cells via mitochondrial dysfunction. Biochem Pharmacol 81: 211-221, 2011.

20. You BR and Park WH: Down-regulation of thioredoxin1 is involved in death of Calu- 6 lung cancer cells treated with suberoyl bishydroxamic acid. J Cell Biochem 117: 1250-1261, 2016.

21. You BR, Shin HR, Han BR, Kim SH and Park WH: Auranofin induces apoptosis and necrosis in HeLa cells via oxidative stress and glutathione depletion. Mol Med Rep 11: 1428-1434, 2015.

22. Park WH: Hydrogen peroxide inhibits the growth of lung cancer cells via the induction of cell death and G1-phase arrest. Oncol Rep 40: 1787-1794, 2018.

23. Park WH, Seol JG, Kim ES, Hyun JM, Jung CW, Lee CC, Kim BK and Lee YY: Arsenic trioxide-mediated growth inhibition in MC/CAR myeloma cells via cell cycle arrest in association with induction of cyclin-dependent kinase inhibitor, p21, and apoptosis. Cancer Res 60: 3065-3071, 2000.

24. Marzano C, Gandin V, Folda A, Scutari G, Bindoli A and Rigobello MP: Inhibition of thioredoxin reductase by auranofin induces apoptosis in cisplatin-resistant human ovarian cancer cells. Free Radic Biol Med 42: 872-881, 2007.

25. Shin DW, Kwon YJ, Ye DJ, Baek HS, Lee JE and Chun YJ: Auranofin suppresses plasminogen activator inhibitor-2 expression through annexin a5 induction in human prostate cancer cells. Biomol Ther (Seoul) 25: 177-185, 2017.

26. Fidyt K, Pastorczak A, Goral A, Szczygiel K, Fendler W, Muchowicz A, Bartlomiejczyk MA, Madzio J, Cyran J, Graczyk-Jarzynka A, et al: Targeting the thioredoxin system as a novel strategy against B-cell acute lymphoblastic leukemia. Mol Oncol 13: 1180-1195, 2019. 
27. Mandal PK, Schneider M, Kölle P, Kuhlencordt P, Förster $H$, Beck H, Bornkamm GW and Conrad M: Loss of thioredoxin reductase 1 renders tumors highly susceptible to pharmacologic glutathione deprivation. Cancer Res 70: 9505-9514, 2010.

28. Wen C, Wang H, Wu X, He L, Zhou Q, Wang F, Chen S, Huang L, Chen J, Wang H, et al: ROS-mediated inactivation of the PI3K/AKT pathway is involved in the antigastric cancer effects of thioredoxin reductase-1 inhibitor chaetocin. Cell Death Dis 10: 809, 2019.
29. Radenkovic F, Holland O, Vanderlelie JJ and Perkins AV: Selective inhibition of endogenous antioxidants with Auranofin causes mitochondrial oxidative stress which can be countered by selenium supplementation. Biochem Pharmacol 146: 42-52, 2017.

30. Mejia EM and Hatch GM: Mitochondrial phospholipids: Role in mitochondrial function. J Bioenerg Biomembr 48: 99-112, 2016. 